\title{
Conformational complexity of morphine and morphinum in the gas phase and in water. A DFT and MP2 study $\dagger$
}

Cite this: RSC Adv., 2014, 4, 24729

\author{
Harald Møllendal, ${ }^{\text {a }}$ David Balcells, ${ }^{a}$ Odile Eisenstein, ${ }^{\mathrm{ab}}$ Linda Syversen $^{\mathrm{c}}$ \\ and Michal Rachel Suissa*c
}

The structural and conformational properties of morphine and protonated morphine (morphinum) in the gas phase and in water solution have been explored with quantum calculations. Fully optimized calculations using the cc-pVTZ basis set, with various methods (MP2, B3LYP, and PBE0) for the species in the gas phase and with B3LYP with simulation of the solvent effect as a continuum with the SMD method were conducted. The study focuses on the determination of the relative energies of the 12 possible conformers that arise from the orientation of the two $\mathrm{OH}$ groups and the equatorial vs. axial position of the methyl group on the nitrogen and the energy barriers that separate these minima. The calculations indicate a preference for conformers having the methyl group equatorial, but corresponding axial conformers are not significantly higher in energy. Only 8 of the 12 possible conformers of gaseous morphine were found to be minima on the potential energy hypersurface. All 12 conformers of morphinum are minima according to MP2 computations. B3LYP/SMD (water) calculations predict the coexistence of 12 conformers for both morphine and morphinum with energy ranges of $17 \mathrm{~kJ} \mathrm{~mol}^{-1}$ for morphine, and as low as $13 \mathrm{~kJ} \mathrm{~mol}^{-1}$ for morphinum. In morphinum, energy differences of less than $8 \mathrm{~kJ}$ $\mathrm{mol}^{-1}$ are computed for 8 conformers, including axial forms. The inversion at nitrogen is calculated to be energetically accessible at room temperature since the activation barrier is less than $30 \mathrm{~kJ} \mathrm{~mol}^{-1}$ in the gas phase and only around $40 \mathrm{~kJ} \mathrm{~mol}^{-1}$ with simulated water solvation. The many conformers within

Received 3rd April 2014 Accepted 22nd May 2014

DOI: $10.1039 / c 4 r a 02992 e$

www.rsc.org/advances a small energy span, the fact that a thermodynamic equilibrium exists between morphine and morphinum in water, and the rapid nitrogen inversion show that morphine and morphinum have a large conformational diversity in water, and thus in the physiological media, which could be a clue to the interaction of this drug with receptors.

\section{Introduction}

Morphine is a highly potent opiate analgesic drug that acts directly on the central nervous system, the peripheral nervous system, and the gastrointestinal tract. It is used as an analgesic (relieves or eliminates pain) and as a narcotic. Morphine has many undesirable side effects such as addiction, constipation,

\footnotetext{
${ }^{a}$ Centre for Theoretical and Computational Chemistry (CTCC), Department of Chemistry, University of Oslo, P. O. Box 1033 Blindern, NO-0315 Oslo, Norway. E-mail: harald.mollendal@kjemi.uio.no; Fax: +47-2285-5674; Tel: +47-2285-5674 ${ }^{b}$ Institut Charles Gerhardt, UMR 5253 CNRS Université Montpellier 2, cc 1501 place E. Bataillon, 34095 Montpellier, France

'Oslo and Akershus University College of Applied Sciences, Pilestredet 50, NO-0167 Oslo, Norway

$\dagger$ Electronic supplementary information (ESI) available: Fully optimized MP2, B3LYP, PBE0, and B3LYP/SMD structures in Cartesian coordinates of all extrema (minima and transition states) and electronic energies obtained using the cc-pVTZ basis set. Tables with relative electronic energies, enthalpies, and Gibbs energies. X-ray and MP2 structures of one conformer of each of morphine and morphinum. See DOI: 10.1039/c4ra02992e
}

abdominal cramps, diarrhoea, vomiting, drowsiness, etc. Properties of this much investigated prototypical opioid have been reviewed extensively. ${ }^{1-12}$

The numerous physiological properties of morphine ultimately depend on the structural (in large part conformational) properties of this compound and on its N-protonated analogue, the morphinum cation, but the relations between structures and functions are still unclear. Furthermore, the diversity of the possible conformations has not yet been fully understood. Studies, such as the present one, should therefore not only lead to a better understanding of the conformational properties of morphine and morphinum, but could help understanding their physiological behaviour and be helpful for the efforts of synthesizing drugs free of unwanted side effects.

Morphine $\left(\mathrm{C}_{17} \mathrm{H}_{19} \mathrm{NO}_{3}\right)$ is composed of five condensed rings (A-E; see Fig. 1) in a rigid quasi diamond-like structure. The labelling of the nitrogen and carbon atoms is shown in Fig. 1. The protonated N17-form morphinum $\left(\mathrm{C}_{17} \mathrm{H}_{20} \mathrm{NO}_{3}{ }^{+}\right)$, is a twoprotic acid with $\mathrm{p} K_{\mathrm{a} 1}=8.31(\mathrm{~N} 17$, ring $\mathrm{D})$ and $\mathrm{p} K_{\mathrm{a} 2}=9.51$ (phenol function, ring A). ${ }^{13}$ At physiological pH (7.365), the 

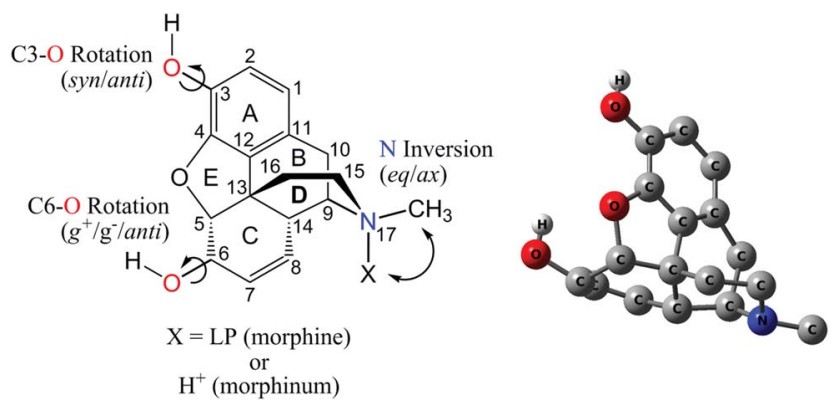

Fig. 1 Morphine and morphinum (left) with atom numbering and the $X$-ray structure of morphine (right). The conformation of morphine found in the $\mathrm{X}$-ray work is denoted $\mathrm{M}_{\text {equ }}-3 a n t i-6 g^{+}$. Hydrogen atoms, other than those of interest for the conformational properties, have been omitted for clarity.

concentration of the morphinum cation is about 9 times larger than that of morphine. At this $\mathrm{pH}$, there is also an insignificant concentration of the phenoxidic anion $\left(\mathrm{C}_{17} \mathrm{H}_{18} \mathrm{NO}_{3}{ }^{-}\right)$, which is therefore not considered further.

The conformational properties of morphine and morphinum are associated with the equatorial and axial positions of the methyl group at $\mathrm{N} 17$, as well as the rotational isomerism about the C3-O and C6-O bonds (Fig. 1). For convenience, the conformers with equatorial and axial methyl group in the morphine and morphinum are denoted as $\mathbf{M}_{\mathrm{equ}}, \mathbf{M}_{\mathrm{ax}}, \mathbf{M}_{\mathrm{equ}}^{+}$, and $\mathrm{M}_{\mathrm{ax}}^{+}$, respectively.

Microwave studies have shown that the phenol OH group lies in the aromatic plane, ${ }^{\mathbf{1 4}}$ which indicates that the dihedral angle $\mathrm{C} 4-\mathrm{C} 3-\mathrm{O}-\mathrm{H}$ is either $\approx 0^{\circ}$ (synperiplanar) or $180^{\circ}$ (antiperiplanar). These two conformations will be noted 3 syn and 3anti. The other hydroxyl group is bonded to a $\mathrm{sp}^{3}$ carbon C6 and it is well established that three rotamer forms could exist. In the antiperiplanar rotamer, the $\mathrm{C} 5-\mathrm{C} 6-\mathrm{O}-\mathrm{H}$ dihedral angle is $\approx 180^{\circ}$, whereas it is about $+60^{\circ}$ in the + synclinal form and $-60^{\circ}$ in the -synclinal conformer. The three rotamer forms of the hydroxyl group at C6 are referred to as 6 anti, $6 g^{+}$, and $6 g^{-}$, respectively. Combining the conformational possibilities for the two $\mathrm{OH}$ groups leads to 6 possible conformers for morphine for a given position of the methyl group at nitrogen. Since this methyl group at N17 can be either equatorial or axial (isomers referred as equatorial or axial in this work), there are 12 possible conformers for morphine. A similar reasoning applies to morphinum, which also has 12 possible conformers.

There are several recent experimental and theoretical investigations of the conformational and structural properties related to morphine and morphinum. The ${ }^{13} \mathrm{C}$ NMR studies show that the equatorial to axial inversion at $\mathrm{N}$ in morphine analogues, codeine and sinomenine, in aprotic solvents is relatively fast with an energy barrier of $25-27 \mathrm{~kJ} \mathrm{~mol}^{-1} \cdot{ }^{15}$ A MM3 calculation of morphine yield a value of $27.6 \mathrm{~kJ} \mathrm{~mol}^{-1},{ }^{16}$ which agree well with the experimental values. ${ }^{15}$

Crystalline morphine exists in two polymorphic forms. ${ }^{17}$ Very recently, the X-ray structure of the stable polymorph was reported. ${ }^{18}$ The heavy-atom skeleton of this compound is drawn in Fig. 1 (right). It has an equatorial methyl group, the hydroxyl group attached to $\mathrm{C} 6$ is engaged in an intramolecular hydrogen bond with the ether oxygen atom of the E ring $\left(6 g^{+}\right.$conformation), and the phenolic $\mathrm{OH}$ group of $\mathrm{C} 3$ has an 3anti conformation and forms an intermolecular hydrogen bond with a neighbour morphine molecule. A recent X-ray determination of the structure of morphine hydrochloride anhydrate ${ }^{19}$ reveals that the morphinum cation has the same conformation as morphine $^{\mathbf{1 8}}$ with one exception, namely, the phenolic $\mathrm{OH}$, which now has a 3 syn conformation forming a second hydrogen bond with oxygen of the E ring.

It is typical that only one conformer is present in the crystalline state of both morphine and its hydrochloride. However, relatively small energy differences are expected between several conformers of morphine and morphinum in the gas phase and in water solution. Unfortunately, no experimental methods are presently able to map the full conformational landscape of such relatively complicated compounds. Consequently, a computational study is an attractive alternative method. Computations with quantum methods restricted to equatorial conformations of morphine have recently been reported for the gas phase, ${ }^{\mathbf{2 0 - 2 2}}$ as well as for aqueous solution. ${ }^{20}$ In this work, state-of-the-art quantum calculations are carried out to investigate the full conformational landscape of morphine and morphinum in the gas phase and in water solution. The study includes calculations for equatorial morphine to a higher methodological level than previously ${ }^{20-22}$ using a choice of several DFT functionals and a wave-function method, MP2. The axial conformations of morphine, not considered in previous works, were included. ${ }^{\mathbf{2 0 - 2 2}}$ This study reveals that axial conformers are not much higher in energies than their equatorial counterparts and should definitely be taken into consideration. No similar calculations have previously been reported for morphinum, the predominating species in water solutions at physiological $\mathrm{pH}$ and the first results are therefore reported herein. The energies of transition states of nitrogen inversion of morphine derivatives reported previously $^{15}$ were obtained in aprotic solvents and do not consider the various individual conformers involved in this motion. This is also the case for the MM3 calculations, which refer to the gas phase. ${ }^{16}$ In the present study, the equatorial to axial transition states of several conformers are calculated for the first time both for the gas phase and water solution and it is shown that solvation modifies the barrier heights.

\section{Computational details}

The calculations were carried out with the Gaussian 09 program $^{23}$ running on the Abel cluster of the University of Oslo. DFT and wave-function based methods were used in order to detect small energy variations originating from weak interactions within certain conformations of morphine/morphinum. For the DFT methods, the B3LYP ${ }^{24,25}$ and PBE0, ${ }^{26}$ functionals were employed. Selected calculations were carried out using the M06-2X $\mathrm{X}^{27}$ functional. The MP2 method was used as a wavefunction based procedure. The influence of the solvent (water) was modelled using the SMD continuum solvation method of Marenich et $a .^{28}$ only in the case of B3LYP calculations. The modeling by a continuum was selected over the introduction of explicit water molecules because it was considered that a large 
number of water molecules would have been needed to treat in an equal manner the solvation of the two $\mathrm{OH}$ and the amine/ ammonium groups. The cc-pVTZ basis set of Peterson and Dunning is used for all atoms and with all methods. ${ }^{29,30}$ All minima, including those obtained in the MP2 and B3LYP/SMD calculations, were fully optimized without any constraints. To explore the potential energy surfaces associated with the rotation of the $\mathrm{OH}$ groups, a large number of relaxed scans were conducted at the B3LYP level in which the position of one of the two $\mathrm{OH}$ groups was varied via a dihedral angle $\left(10^{\circ}\right.$ steps $)$ defined relative to the carbon skeleton, while all other structural parameters are optimized. The information obtained from this exploration was used for carrying out full optimization of minima and transition states. The nature of each extremum (minimum or transition state) was assigned by analytical calculations of frequencies in the case of the B3LYP and PBE0 functionals. The MP2 energies of the transition states were carried out as single point calculations on B3LYP geometries. The Gibbs energies and enthalpies were calculated from the harmonic approximation of frequencies at $298 \mathrm{~K}$ and $P=1 \mathrm{~atm}$ only with the B3LYP and PBE0 functionals. The trends in electronic energies and Gibbs energies and enthalpies are similar and for this reason the electronic energies available for all methods are used to present the results.

\section{Results and discussion}

\subsection{Rotational energy profiles of morphine and morphinum}

A series of B3LYP scans about either the C3-O or the C6-O bond were undertaken for the isomer with the equatorial and axial methyl group at nitrogen, $\mathbf{M}_{\mathrm{equ}}$ and $\mathrm{M}_{\mathrm{ax}}$, respectively. The corresponding calculations were carried out for the morphinum form $\left(\mathrm{M}_{\mathrm{equ}}^{+}\right.$, and $\left.\mathrm{M}_{\mathrm{ax}}^{+}\right)$. This first set of calculations was carried out for systems in the gas phase. The phenol $\mathrm{OH}$ was held in either the 3syn or 3anti conformation for the study of the rotation about the $\mathrm{C} 6-\mathrm{O}$ bond ( $\omega=$ dihedral angle $\mathrm{C} 5-\mathrm{C} 8-\mathrm{O}-\mathrm{H})$. Likewise, the rotation about the $\mathrm{C} 3-\mathrm{O}$ bond was performed while holding the alcohol $\mathrm{C} 6-\mathrm{OH}$ in either the $6 g^{+}, 6 g^{-}$, or in the 6anti position.

A typical example is the potential curve of isolated $\mathbf{M}_{\text {equ }}$ (black line in Fig. 2) for the rotation about the C6-O bond $(\omega)$ with phenolic $\mathrm{OH}$ in the $3 \operatorname{syn}$ conformation $\left(\omega^{\prime}=0^{\circ}\right)$. There are only two minima on this curve, one corresponding to $6 g^{+}\left(\omega \approx 50^{\circ}\right)$ and the other one to 6 anti $\left(\omega \approx 180^{\circ}\right), 15.9 \mathrm{~kJ} \mathrm{~mol}^{-1}$ higher in energy than $6 g^{+}$. Interestingly, the expected $g^{-}$-minimum for $\omega \approx$ $300^{\circ}\left(-60^{\circ}\right)$ is missing. However, an inflexion in the curve is seen for $\omega \approx 300^{\circ}$. The maxima for $\omega$ appear at approximately 130 and $250^{\circ}$. Their energies are 18.9 and $18.0 \mathrm{~kJ} \mathrm{~mol}^{-1}$ above $6 g^{+}$.

The corresponding B3LYP/SMD energies obtained for $\mathrm{M}_{\mathrm{equ}}$ in water are shown as the red curve in Fig. 2. Comparison with the calculations for $\mathbf{M}_{\text {equ }}$ in the gas phase (black curve) shows that the solvation modifies the conformational properties significantly. The energies of the maxima for $\omega \approx 130^{\circ}(10.5 \mathrm{~kJ}$ $\mathrm{mol}^{-1}$ above the energy of $\left.6 g^{+}\right)$, at ca. $240^{\circ}\left(8.3 \mathrm{~kJ} \mathrm{~mol}^{-1}\right)$, and at about $340^{\circ}\left(7.8 \mathrm{~kJ} \mathrm{~mol}^{-1}\right)$, are significantly lower than for the gas phase (black curve). $6 g^{-}$is now suggested to be a possible secondary minimum in contrast to the gas-phase results.

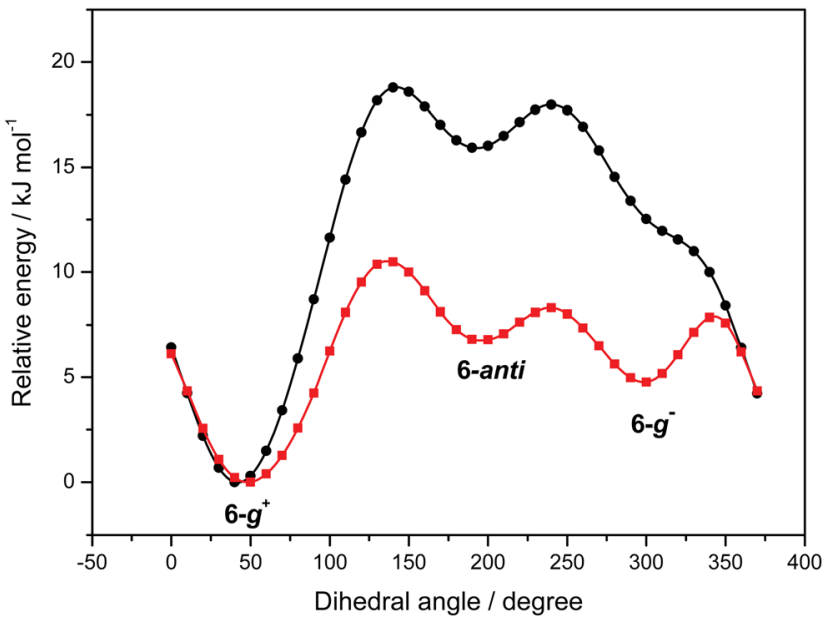

Fig. 2 Potential energy (B3LYP) curves for rotation about the $\mathrm{C6}-\mathrm{O}$ bond $(\omega)$ of equatorial morphine with $\mathrm{C} 4-\mathrm{C} 3-\mathrm{O}-\mathrm{H}$ dihedral angle $\left(\omega^{\prime}=0^{\circ}\right)$ in the 3 syn position. The black curve refers to calculations in gas phase and the red curve to calculations including the solvation effect (water).

The B3LYP energy for rotation about $\mathrm{C} 3-\mathrm{O}$ bond of $\mathrm{M}_{\mathrm{equ}}$ $\left(\omega \omega^{\prime}=\mathrm{C} 4-\mathrm{C} 3-\mathrm{O}-\mathrm{H}\right)$ with the $\mathrm{C}_{5} \mathrm{C}_{6} \mathrm{OH}$ in the $6 g^{+}$conformation is shown as the black curve in Fig. 3. There are the two expected minima on this potential energy curve corresponding to $3 s y n$ and 3 anti, respectively, with $3 s y n$ being more stable than $3 a n t i$ by $6.7 \mathrm{~kJ} \mathrm{~mol}^{-1}$. The two maxima are located at $\omega^{\prime} \approx 100$ and $270^{\circ}$, at 16.8 and $17.8 \mathrm{~kJ} \mathrm{~mol}^{-1}$ above the 3 syn minimum. This is in good agreement with the experimental value of the barrier to internal rotation of the $\mathrm{OH}$ group of phenol, which is 16.3(6) kJ $\mathrm{mol}^{-1} \cdot{ }^{31}$ Related computations for $\mathrm{M}_{\mathrm{equ}}$ in water with the alcohol $\mathrm{OH}$ group held in the $6 g^{+}$position resulted in the curve shown in red in Fig. 3. The solvent lowers the energy of the

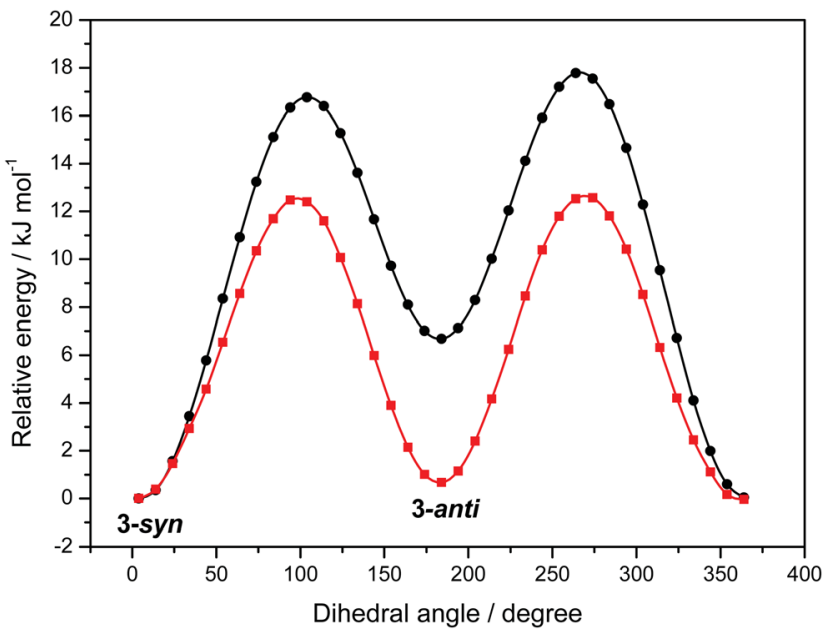

Fig. 3 Potential energy (B3LYP) curves for rotation about the C3-O bond $\left(\omega^{\prime}\right)$ of equatorial morphine with $\mathrm{C} 5-\mathrm{C} 6-\mathrm{O}-\mathrm{H}$ dihedral angle $\left(\omega=+60^{\circ}\right)$ in the $6 g^{+}$position. The black curve refers to calculations in gas phase and the red curve to calculations including the solvation effect (water). 
maxima (12.5 and $12.6 \mathrm{~kJ} \mathrm{~mol}^{-1}$ ) and essentially equalizes the energy of 3syn and 3anti (difference of $0.7 \mathrm{~kJ} \mathrm{~mol}^{-1}$ ).

Related curves for morphinum are shown in Fig. 4 (rotation about the C6-O bond described by $\omega$ ) and Fig. 5 (rotation about the C3-O bond described by $\omega^{\prime}$ ), where the black and red graphs represent the gas phase and the water solution, respectively. The variations of the energies as a function of $\omega$ or $\omega^{\prime}$ are smaller when the solvent effect is included. An exception appears for values of $\omega$ larger than $320^{\circ}$, where the values of energies including solvation are higher (Fig. 4). The maxima of the black curve of Fig. 4 (gas phase) occur at $\omega=140,240$, and $340^{\circ}$ with energies $12.8,10.0$, and $6.6 \mathrm{~kJ} \mathrm{~mol}^{-1}$ higher than that of the $6 g^{+}$minimum. The maxima of the water-solution graph (red curve) occur nearly for the same values of $\omega$ as for the gas phase with energy values that are $10.6,8.2$, and $7.8 \mathrm{~kJ} \mathrm{~mol}^{-1}$ higher than that of the $6 g^{+}$minimum. The potential energy curve for rotation about C3-O (Fig. 5) shows that the gas barrier heights are about 16.9 and $18.7 \mathrm{~kJ} \mathrm{~mol}^{-1}$ for $\omega^{\prime}=100$ and $270^{\circ}$, respectively, relative to the 3 syn minimum. The corresponding values are $12.5\left(93^{\circ}\right)$ and $12.6 \mathrm{~kJ} \mathrm{~mol}^{-1}\left(273^{\circ}\right)$ for water solution.

\subsection{Optimized structures of morphine and morphinum conformers}

Many additional scans similar to those described above reveal that several rotamers exist within a relatively small energy span and that the energy barriers separating them are relatively low. This information was used to carry out a full optimization of minima at the B3LYP, B3LYP/SMD, PBE0 and MP2 levels. As mentioned in the Computational details section the vibrational frequencies were calculated only with the B3LYP and PBE0 methods. The relative energies of minima are listed in Tables 1 and 2 for morphine and Tables 3 and 4 for morphinum. The Cartesian coordinates of all extrema are listed in the ESI, Tables 1S-90S. $\dagger$ Enthalpies and Gibbs energies calculated using the

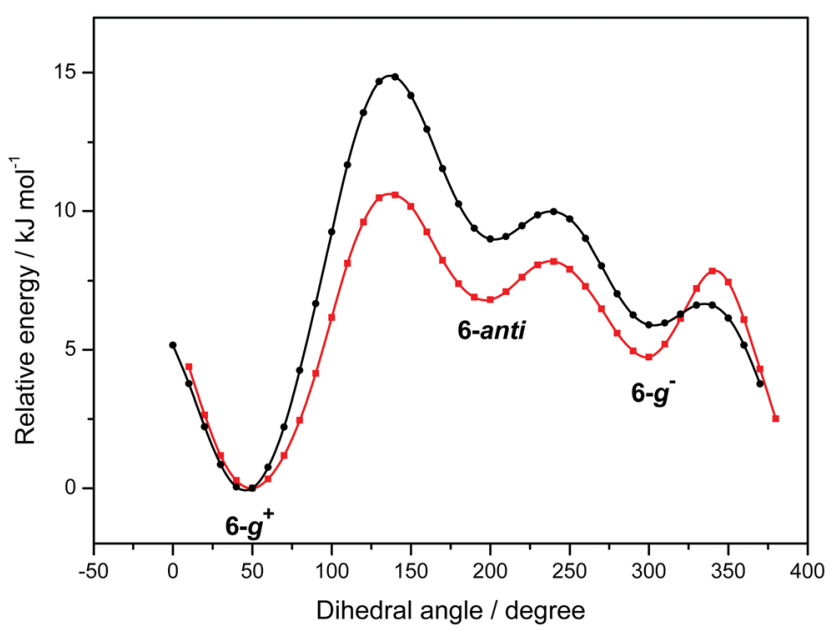

Fig. 4 Potential energy (B3LYP) curves for rotation about the C6-O bond $(\omega)$ of equatorial morphinum with $\mathrm{C} 4-\mathrm{C} 3-\mathrm{O}-\mathrm{H}$ dihedral angle $\left(\omega^{\prime}=0^{\circ}\right)$ in the 3syn position. The black curve refers to calculations in gas phase and the red curve to calculations including the solvation effect (water).

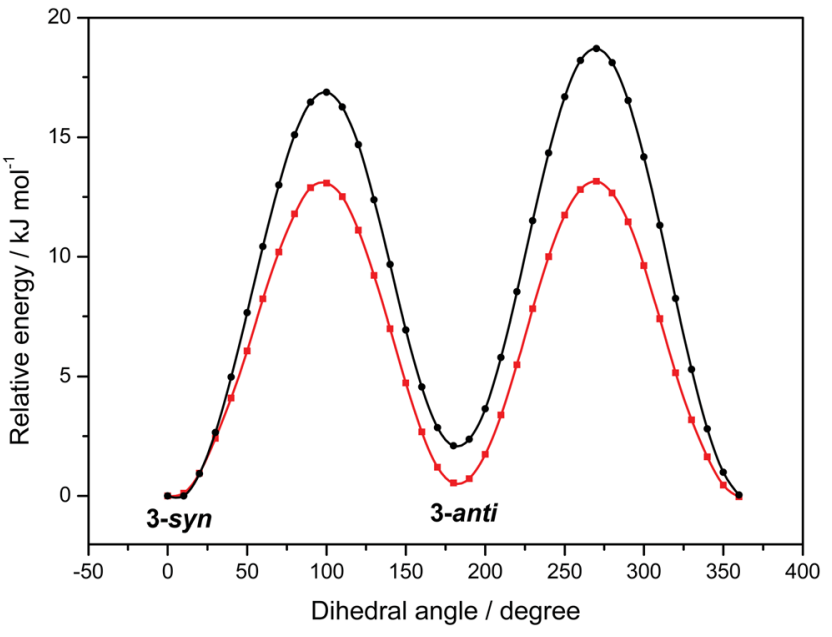

Fig. 5 Potential energy (B3LYP) curves for rotation about the C3-O bond $\left(\omega^{\prime}\right)$ of equatorial morphinum with $\mathrm{C} 5-\mathrm{C} 6-\mathrm{O}-\mathrm{H}$ dihedral angle $(\omega=+60)$ in the $6 g^{+}$position. The black curve refers to calculations in gas phase and the red curve to calculations including the solvation effect (water) by a continuum (SMD) method.

Table 1 B3LYP relative energies ${ }^{a, b}$ and dipole moments of conformers of morphine in the gas phase and in water solution

\begin{tabular}{|c|c|c|c|c|}
\hline \multirow[b]{2}{*}{ Method } & \multicolumn{2}{|c|}{ B3LYP (gas) } & \multicolumn{2}{|c|}{ B3LYP/SMD (water) } \\
\hline & $\begin{array}{l}\Delta E / \mathrm{kJ} \\
\mathrm{mol}^{-1}\end{array}$ & $\begin{array}{l}\text { Dipole } \\
\text { moment/D }\end{array}$ & $\begin{array}{l}\Delta E / \mathrm{kJ} \\
\mathrm{mol}^{-1}\end{array}$ & $\begin{array}{l}\text { Dipole } \\
\text { moment/D }\end{array}$ \\
\hline $\mathrm{M}_{\mathrm{ax}}-3$ anti-6anti & 39.6 & 2.73 & 16.6 & 4.75 \\
\hline $\mathrm{M}_{\mathrm{equ}}-3 a n t i-6 a n t i$ & 29.4 & 2.85 & 8.1 & 4.95 \\
\hline $\mathrm{M}_{\mathrm{ax}}-3 s y n-6 a n t i$ & 26.3 & 4.07 & 15.4 & 6.67 \\
\hline 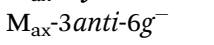 & $-^{c}$ & & 14.7 & 3.67 \\
\hline $\mathbf{M}_{\mathrm{ax}}-3 s y n-6 g^{-}$ & $-^{c}$ & & 13.6 & 6.22 \\
\hline $\mathrm{M}_{\mathrm{ax}}-3$ anti- $6 g^{+}$ & 16.8 & 2.72 & 9.1 & 3.87 \\
\hline $\mathrm{M}_{\mathrm{equ}}-3 s y n-6 a n t i$ & 15.9 & 3.61 & 6.7 & 5.60 \\
\hline $\mathrm{M}_{\mathrm{ax}}-3 s y n-6 g^{+}$ & 10.3 & 2.65 & 8.6 & 4.09 \\
\hline 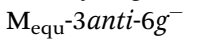 & $-^{c}$ & & 6.3 & 3.94 \\
\hline $\mathrm{M}_{\mathrm{equ}}-3 s y n-6 g^{-}$ & $-^{c}$ & & 5.0 & 5.13 \\
\hline $\mathrm{M}_{\mathrm{equ}}-3$ anti- $6 g^{+}$ & 6.6 & 3.48 & 0.8 & 5.32 \\
\hline $\mathrm{M}_{\mathrm{equ}}-3 s y n-6 g^{+}$ & 0.0 & 2.73 & 0.0 & 3.94 \\
\hline
\end{tabular}

${ }^{a}$ The cc-pVTZ basis set was used in all calculations; see text. ${ }^{b}$ Absolute energies and structures in Cartesian coordinates are given in the ESI. $\dagger$ ${ }^{c}$ This form was not found as a minimum on the conformational energy hypersurface; see text.

harmonic vibrational frequencies obtained in B3LYP and PBE0 calculations are listed in Tables 91S and 92S of the ESI. $\dagger$ MP2 bond distances are given in Table 93S of the ESI $\dagger$ for the conformers of morphine and morphinum observed in the solidstate by the X-ray diffraction studies. ${ }^{18,19}$ Dipole moments could play a role for the relative stability in water and are therefore included in Tables 1 and 3. Selected conformers of equatorial and axial morphine and their morphinum counterparts are shown in Fig. 6.

Inspection of the structures of the many conformers reveals few unusual or unexpected features. The $\mathrm{C} 4 \mathrm{C} 3 \mathrm{OH}\left(\omega^{\prime}\right)$ and $\mathrm{C}_{5} \mathrm{C}_{6} \mathrm{OH}(\omega)$ dihedral angles are the parameters to consider to 
Table 2 MP2 and PBEO relative energies ${ }^{a, b}$ of conformers of morphine in the gas phase

\begin{tabular}{|c|c|c|}
\hline Method & MP2 & PBE0 \\
\hline Relative energy & $\Delta E / \mathrm{kJ} \mathrm{mol}^{-1}$ & $\Delta E / \mathrm{kJ} \mathrm{mol}^{-1}$ \\
\hline $\mathbf{M}_{\mathrm{ax}}-3 a n t i-6 a n t i$ & 37.6 & 38.5 \\
\hline $\mathrm{M}_{\mathrm{equ}}-3$ anti-6anti & 29.4 & 28.9 \\
\hline $\mathrm{M}_{\mathrm{ax}}-3 s y n-6 a n t i$ & 23.9 & 25.5 \\
\hline $\mathbf{M}_{\mathrm{ax}}-3$ anti- $6 g^{-}$ & $-^{c}$ & $-{ }^{c}$ \\
\hline $\mathbf{M}_{\mathrm{ax}}-3 s y n-6 g^{-}$ & $-^{c}$ & $-^{c}$ \\
\hline $\mathrm{M}_{\mathrm{ax}}-3$ anti- $6 g^{+}$ & 14.8 & 15.7 \\
\hline $\mathrm{M}_{\mathrm{equ}}-3 \operatorname{syn}-6$ anti & 15.4 & 15.8 \\
\hline $\mathbf{M}_{\mathrm{ax}}-3 \operatorname{syn}-6 g^{+}$ & 8.3 & 9.8 \\
\hline $\mathrm{M}_{\mathrm{equ}}-3$ anti- $6 g^{-}$ & $-^{c}$ & $-^{c}$ \\
\hline $\mathrm{M}_{\mathrm{equ}}{ }^{-3 s y n}-6 g^{-}$ & $-^{c}$ & $-^{c}$ \\
\hline $\mathrm{M}_{\mathrm{equ}}-3$ anti- $6 g^{+}$ & 6.7 & 6.1 \\
\hline $\mathrm{M}_{\mathrm{equ}}-3 s y n-6 g^{+}$ & 0.0 & 0.0 \\
\hline
\end{tabular}

${ }^{a}$ The cc-pVTZ basis set was used in all calculations; see text. ${ }^{b}$ Absolute energies and structures in Cartesian coordinates are given in the ESI. $\dagger$ ${ }^{c}$ This form was not found as a minimum on the conformational energy hypersurface; see text.

Table 3 B3LYP relative energies ${ }^{a, b}$ and dipole moments conformers of morphinum in the gas phase and in water solution

\begin{tabular}{|c|c|c|c|c|}
\hline \multirow[b]{2}{*}{ Method } & \multicolumn{2}{|c|}{ B3LYP (gas) } & \multicolumn{2}{|c|}{ B3LYP/SMD (water) } \\
\hline & $\begin{array}{l}\Delta E / \mathrm{kJ} \\
\mathrm{mol}^{-1}\end{array}$ & $\begin{array}{l}\text { Dipole } \\
\text { moment/D }\end{array}$ & $\begin{array}{l}\Delta E / \mathrm{kJ} \\
\mathrm{mol}^{-1}\end{array}$ & $\begin{array}{l}\text { Dipole } \\
\text { moment/D }\end{array}$ \\
\hline $\mathrm{M}_{\mathrm{ax}}^{+}-3$ anti-6anti & 23.6 & 11.59 & 13.2 & 17.92 \\
\hline $\mathrm{M}_{\mathrm{equ}}^{+}-3 a n t i-6 a n t i$ & 18.0 & 11.63 & 7.7 & 17.90 \\
\hline $\mathrm{M}_{\mathrm{ax}}^{+}-3 s y n-6 a n t i$ & 14.5 & 12.28 & 12.3 & 18.68 \\
\hline $\mathrm{M}_{\mathrm{ax}}^{+}-3$ anti- $6 g^{-}$ & $-^{c}$ & & 11.3 & 17.71 \\
\hline $\mathrm{M}_{\mathrm{ax}}^{+}-3 s y n-6 g^{-}$ & 11.5 & 11.88 & 10.6 & 18.58 \\
\hline $\mathrm{M}_{\mathrm{equ}}^{+}-3 s y n-6 a n t i$ & 9.0 & 12.30 & 6.5 & 18.70 \\
\hline $\mathrm{M}_{\mathrm{ax}}^{+}-3$ anti- $6 g^{+}$ & 7.7 & 11.99 & 5.9 & 17.73 \\
\hline $\mathrm{M}_{\mathrm{equ}}^{+}-3$ anti- $6 g^{-}$ & $-^{c}$ & & 5.9 & 17.72 \\
\hline $\mathrm{M}_{\mathrm{equ}}^{+}-3 s y n-6 g^{-}$ & 5.9 & 11.97 & 4.8 & 18.63 \\
\hline $\mathrm{M}_{\mathrm{ax}}^{+}-3 s y n-6 g^{+}$ & 5.6 & 12.23 & 5.6 & 17.91 \\
\hline $\mathrm{M}_{\mathrm{equ}}^{+}-3$ anti- $6 g^{+}$ & 2.2 & 12.02 & 0.6 & 17.59 \\
\hline $\mathrm{M}_{\mathrm{equ}}^{+}-3 s y n-6 g^{+}$ & 0.0 & 12.23 & 0.0 & 17.86 \\
\hline
\end{tabular}

describe the conformational properties. The values of $\omega^{\prime}$ and $\omega$ at the MP2 level are +5 and $+42^{\circ}$, respectively, in $\mathrm{M}_{\text {equ }}-3 s y n-6 g^{+}$, while +6 and $+45^{\circ}$ were obtained for its morphinum analogue $\mathrm{M}_{\mathrm{equ}}^{+}-3$ syn- $6 g^{+}$. The significant deviation from $+60^{\circ}$ for $\omega$ is probably caused largely by hydrogen bonding with the oxygen atom of the E ring (Fig. 1). A rotation of the $\omega$ dihedral angle to less than $+60^{\circ}$ brings the hydrogen atom of the hydroxyl group and the oxygen atom of the $\mathrm{E}$ ring into closer proximity strengthening the intramolecular hydrogen bond. Typical values of $\omega$ for 6 anti conformers were about $-170^{\circ}$, while the $g^{-}$ angles of morphinum conformers were approximately $-60^{\circ}$.

Several interesting features emerge from Tables 1-4. The isomers with the equatorial methyl group at nitrogen,
Table 4 MP2 and PBEO relative energies ${ }^{a, b}$ of conformers of morphinum in the gas phase

\begin{tabular}{|c|c|c|}
\hline Method & MP2 & PBE0 \\
\hline Relative energy & $\Delta E / \mathrm{kJ} \mathrm{mol}^{-1}$ & $\Delta E / \mathrm{kJ} \mathrm{mol}^{-1}$ \\
\hline $\mathrm{M}_{\mathrm{ax}}^{+}-3$ anti-6anti & 21.9 & 23.2 \\
\hline $\mathrm{M}_{\mathrm{equ}}^{+}-3$ anti-6anti & 18.0 & 17.6 \\
\hline $\mathbf{M}_{\mathrm{ax}}^{+}-3 s y n-6 a n t i$ & 12.5 & 14.5 \\
\hline $\mathrm{M}_{\mathrm{ax}}^{+}-3$ anti- $6 g^{-}$ & 9.9 & $-^{c}$ \\
\hline $\mathrm{M}_{\mathrm{ax}}^{+}-3 s y n-6 g^{-}$ & 9.3 & 12.0 \\
\hline $\mathrm{M}_{\mathrm{equ}}^{+}-3$ syn-6anti & 8.7 & 9.0 \\
\hline $\mathrm{M}_{\mathrm{ax}}^{+}-3$ anti- $6 g^{+}$ & 5.9 & 7.8 \\
\hline $\mathrm{M}_{\mathrm{equ}}^{+}-3 a n t i-6 g^{-}$ & 5.3 & $-^{c}$ \\
\hline $\mathbf{M}_{\text {equ }}^{+}-3 s y n-6 g^{-}$ & 4.6 & 6.3 \\
\hline $\mathrm{M}_{\mathrm{ax}}^{+}-3 s y n-6 g^{+}$ & 3.9 & 5.6 \\
\hline $\mathrm{M}_{\mathrm{equ}}^{+}-3$ anti- $6 g^{+}$ & 2.1 & 1.6 \\
\hline $\mathrm{M}_{\mathrm{equ}}^{+}-3 s y n-6 g^{+}$ & 0.0 & 0.0 \\
\hline
\end{tabular}

${ }^{a}$ The cc-pVTZ basis set was used in all calculations; see text. ${ }^{b}$ Absolute energies and structures in Cartesian coordinates are given in the ESI. $\dagger$ ${ }^{c}$ This form was not found as a minimum on the conformational energy hypersurface; see text.
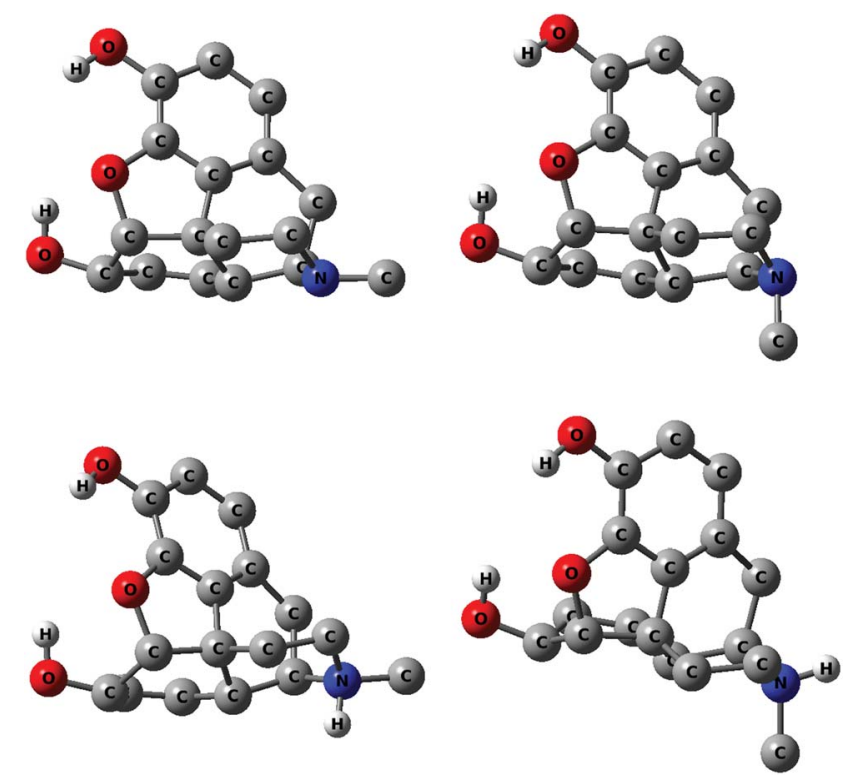

Fig. 6 The two lowest energy conformers both in the gas phase and in water solution of equatorial (upper left) and axial morphine (upper right) and the corresponding two conformers with equatorial methyl group (lower left) and axial methyl group in morphinum (lower right). Hydrogen atoms attached to carbon atoms have been omitted for clarity.

$\mathrm{M}_{\mathrm{equ}}-3 \operatorname{syn}-6 g^{+}$and $\mathrm{M}_{\mathrm{equ}}^{+}-3 \operatorname{syn}-6 g^{+}$, indeed have the lowest energies in both the gas phase and in water presumably because the phenol and alcohol hydroxyl groups are involved in bifurcated intramolecular hydrogen bonding with the oxygen atom of the E ring (Fig. 1). The non-bonded hydrogen to oxygen distance, taken at the MP2 level, is $2.10 \AA$ for the alcohol hydrogen case, and $2.45 \AA$ for the phenol part, which are less than the sum of the Pauling van der Waals radii of 
oxygen and hydrogen, $2.60 \AA^{32}$ The first hydrogen bonds is therefore of intermediate strength, while that with the phenol group is probably marginal. An estimate of the combined strength of these two intramolecular hydrogen bonds may be obtained by comparing the MP2 internal energies of $\mathrm{M}_{\mathrm{equ}}$ $3 s y n-6 g^{+}$and $\mathrm{M}_{\text {equ }}-3$ anti-6anti, which differ by $29.4 \mathrm{~kJ} \mathrm{~mol}^{-1}$ (Table 1). The energies of the corresponding isomers with axial methyl group differ by $29.3 \mathrm{~kJ} \mathrm{~mol}^{-1}$, which indicates that the position of the methyl group at $\mathrm{N}$ has no influence.

No $g^{-}$forms were located for gaseous morphine both by B3LYP, PBE0, and MP2 methods (Tables 1 and 2). Additional attempts using the M06-2X method ${ }^{27,33}$ gave the same results. In most cases, hypothetical $g^{-}$forms used as the starting point converged to the corresponding $g^{+}$conformers. It is therefore most likely that $g^{-}$forms do not exist for morphine in the gas phase. One possible rational is that the interatomic distance between the hydroxyl hydrogen atom and the hydrogen atom at C6 of $2.30 \AA$ is marginally below $2.40 \AA$, which is the sum of the vdW radii of two hydrogen atoms. ${ }^{34}$ Repulsion between the lone pairs of the alcohol oxygen atom and the oxygen atom of the $\mathrm{E}$ ring may also contribute. However, other factors could be responsible since in the case of morphinum calculated in the gas phase, all $g^{-}$rotamers are found as minima at the MP2 level. These results are method-dependent since $\mathrm{M}_{\mathrm{equ}}^{+}-3$ anti- $6 g^{-}$and $\mathrm{M}_{\mathrm{ax}}^{+}-3$ anti- $6 g^{-}$are not found as minima with the DFT methods (Tables 3 and 4). A reason for this could be that dispersion forces, which are treated differently at the DFT and MP2 levels, play a key role in the weak interactions stabilizing these conformers.

The gas-phase energy differences obtained with two different functionals and the MP2 method are remarkably similar, apart from the problematic $g^{-}$conformers discussed above. This validates the energetic pattern found for most conformers with the exceptions for some of the $g^{-}$forms.

The present calculations point out that the number of conformers and their relative order in energies for morphine and morphinum are different in gas phase and in water solution. In addition, all twelve minima are closer in energy in water solution, (energy span of less than $17 \mathrm{~kJ} \mathrm{~mol}^{-1}$ for morphine and $13 \mathrm{~kJ} \mathrm{~mol}^{-1}$ for morphinum) suggesting that they all could be present in a physiological environment. This is in particular the case of the two lowest isomers, $\mathrm{M}_{\mathrm{equ}}-3 s y n-6 g^{+}$and $\mathrm{M}_{\text {equ}}{ }^{-}$ 3 anti $-6 g^{+}$, which are essentially at the same energy in water. These results suggest that the $\mathrm{OH}$ groups can assume more orientations in aqueous media than in the gas phase. Similar results have been found in a theoretical study of the effect of water on competing intra- and intermolecular hydrogen bonds of selected alcohols and phenols. ${ }^{35}$

The four $g^{-}$conformers of morphine, which are not energy minima in gas phase, become minima in water solution. In addition, $\mathrm{M}_{\mathrm{equ}}-3$ anti-6anti which is $29 \mathrm{~kJ} \mathrm{~mol}^{-1}$ in the gas phase above the global minimum $\mathrm{M}_{\mathrm{equ}}-3 s y n-6 g^{+}$, is only $8.1 \mathrm{~kJ} \mathrm{~mol}^{-1}$ above it in water solution. The polarity of water decreases the intramolecular $\mathrm{H}$ bonds and if water was explicitly represented it would mean that the two $\mathrm{OH}$ groups in $\mathrm{M}_{\text {equ }}-3$ anti-6anti could get involved in $\mathrm{H}$ bonding with water. A similar result is found for the corresponding morphinum pair (Table 3).
Tables 1 and 2 reveal that all computational methods show that the energy differences between corresponding axial and equatorial conformers of morphine are fairly constant $(8-10 \mathrm{~kJ}$ $\mathrm{mol}^{-1}$ ) both in gas phase and in water solution. The same is seen for morphinum (Tables 2 and 3), but the energy differences are somewhat smaller $\left(5-8 \mathrm{~kJ} \mathrm{~mol}^{-1}\right)$. These comparatively small energy differences imply that axial forms contribute significantly to the equilibrium conformational mixture of morphine and morphinum.

In order to get further insight into why the water solution modifies the difference in energy between the isomers the way it does, the dipole moments of the molecules in their various conformations are reported in Tables 1 and 3. However, there is apparently no parallel between the magnitude in the dipole moment and the modification of the energy differences between the conformers and the magnitude of the dipole moment will not be considered further.

Baranska and Kaczor ${ }^{21}$ have carried out a search of the conformational space of morphine in the gas phase at the B3LYP level with an extended basis set and identified only four stable conformers. Rincón et al. ${ }^{20}$ carried out a B3LYP(6$31 \mathrm{G})(\mathrm{d}, \mathrm{p})$ study of morphinum but also explored the conformational space only in the gas phase. This limited exploration of the conformational space followed by single point PCM calculations resulted in the identification of only 3 conformers out of the 12 identified in this work. Thus, this work shows that many more conformations of morphine and specially morphinum are accessible than earlier found, especially when aqueous solvation is included.

\subsection{Transition states of N17 inversion}

The energies of the transition states of inversion at nitrogen, which is associated with an equatorial to axial site change of the N17 methyl group in morphine are crucial for the conformational flexibility. There is no similar process in morphinum where the N17 lone pair is protonated.

The transition states for $\mathrm{N}$ inversion were located at the B3LYP level for the preferred morphine conformers $\left(3 s y n-6 g^{+}\right.$, 3 anti- $6 g^{+}, 3 s y n-6 a n t i$, and $\left.3 a n t i-6 a n t i\right)$ in the gas phase with energy of 27.6, 27.6, 28.1, and $28.1 \mathrm{~kJ} \mathrm{~mol}^{-1}$ above the corresponding stable equatorial form. At the MP2/B3LYP level, the corresponding values are $36.9,36.9,37.3$, and $37.3 \mathrm{~kJ} \mathrm{~mol}^{-1}$. B3LYP/SMD calculations of the transition states were also performed for all six morphine species $3 s y n-6 g^{+}, 3 a n t i-6 g^{+}$, $3 s y n-6 a n t i, 3 a n t i-6 a n t i, 3 s y n-6 g^{-}$, and $3 a n t i-6 g^{-}$. The transition-state energies relative to the corresponding equatorial conformers were $41.1,40.1,41.5,41.0,39.6$, and $40.9 \mathrm{~kJ}$ $\mathrm{mol}^{-1}$, respectively. As expected, a polar solvent increased the energy barrier for $\mathrm{N}$ inversion. However, even in water the energy barriers for inversion at $\mathrm{N}$ is small and a rapid conversion between the equatorial and axial conformers should occur at room temperature. These results are in agreement with the experimental value of $25-27 \mathrm{~kJ} \mathrm{~mol}^{-1}$ reported for the morphine derivatives codeine and sinomenine in aprotic solvents, ${ }^{15}$ and the MM3 barrier of morphine $\left(27.6 \mathrm{~kJ} \mathrm{~mol}^{-1}\right){ }^{16}$ 


\section{Conclusions}

These calculations show that the two $\mathrm{OH}$ groups in morphine and morphinum can take a large number of orientations especially in water solution where many isomers are almost at the same energy. The calculations also show that nitrogen inversion is not energetically difficult and that exchange between the forms where the methyl is equatorial and axial should not be excluded. The differences in energy between the various isomers are even smaller in morphinum. This large conformational diversity in water, and thus in the physiological media, could be a clue to the interaction of this drug with receptors.

\section{Acknowledgements}

The authors thank Anne Horn for her skilful assistance. This work has been supported by the Research Council of Norway through a Centre of Excellence Grant (Grant no. 179568/V30). It has also received support from the Norwegian Supercomputing Program (NOTUR) through a grant of computer time (Grant no. NN4654K). OE and DB thank the CTCC for adjunct professor and researcher positions, respectively. MRS thanks the Faculty of Health Sciences, Pharmacy, Oslo and Akershus University College of Applied Sciences (Grant no. 13175).

\section{References}

1 M. Filizola and L. A. Devi, Nature, 2012, 485, 314-317.

2 H. Ueda and M. Ueda, Front. Biosci., Landmark Ed., 2009, 14, 5260-5272.

3 G. M. Rishton, Am. J. Cardiol., 2008, 101, 43D-49D.

4 J. Mika, Pharmacol. Rep., 2008, 60, 297-307.

5 A. A. Somogyi, D. T. Barratt and J. K. Coller, Clin. Pharmacol. Ther., 2007, 81, 429-444.

6 L. Martini and J. L. Whistler, Curr. Opin. Neurobiol., 2007, 17, 556-564.

7 B. H. Novak, T. Hudlicky, J. W. Reed, J. Mulzer and D. Trauner, Curr. Org. Chem., 2000, 4, 343-362.

8 T. Hudlicky, G. Butora, S. P. Fearnley, A. G. Gum and M. R. Stabile, Stud. Nat. Prod. Chem., 1996, 18, 43.

9 C. Stein, M. Schaefer and H. Machelska, Nat. Med., 2003, 9, 1003-1008.

10 A. M. Trescot, S. Helm, H. Hansen, R. Benyamin, S. E. Glaser, R. Adlaka, S. Patel and L. Manchikanti, Pain Physician, 2008, 11, S5-S62.

11 R. Benyamin, A. M. Trescot, S. Datta, R. Buenaventura, R. Adlaka, N. Sehgal, S. E. Glaser and R. Vallejo, Pain Physician, 2008, 11, S105-S120.

12 F. A. Casy and R. T. Parfitt, Opioid Analgetics: Chemistry and Receptors, Plenum Press, New York, 1986.

13 G. Schill and K. Gustavii, Acta Pharm. Suec., 1964, 1, 24-35.

14 N. W. Larsen, E. Mathier, A. Bauder and H. H. Guenthard, J. Mol. Spectrosc., 1973, 47, 183-188.
15 A. M. Belostotskii, Z. Goren and H. E. Gottlieb, J. Nat. Prod., 2004, 67, 1842-1849.

16 A. M. Belostotskii, H. E. Gottlieb and M. Shokhen, J. Org. Chem., 2002, 67, 9257-9266.

17 L. Kofler, Pharm. Monatsh., 1933, 14, 220-222.

18 T. Gelbrich, D. E. Braun and U. J. Griesser, Acta Crystallogr., Sect. E: Struct. Rep. Online, 2013, 69, o2.

19 T. Gelbrich, D. E. Braun and U. J. Griesser, Acta Crystallogr., Sect. E: Struct. Rep. Online, 2012, 68, o3358-03359.

20 D. A. Rincón, M. N. D. S. Cordeiro and R. A. Mosquera, Int. J. Quantum Chem., 2010, 110, 2472-2482.

21 M. Baranska and A. Kaczor, J. Raman Spectrosc., 2012, 43, 102-107.

22 J. M. P. J. Garrido, M. P. M. Marques, A. M. S. Silva, T. R. A. Macedo, A. M. Oliveira-Brett and F. Borges, Anal. Bioanal. Chem., 2007, 388, 1799-1808.

23 M. J. Frisch, G. W. Trucks, H. B. Schlegel, G. E. Scuseria, M. A. Robb, J. R. Cheeseman, G. Scalmani, V. Barone, B. Mennucci, G. A. Petersson, H. Nakatsuji, M. Caricato, X. Li, H. P. Hratchian, A. F. Izmaylov, J. Bloino, G. Zheng, J. L. Sonnenberg, M. Hada, M. Ehara, R. Fukuda, J. Hasegawa, M. Ishida, T. Nakajima, Y. Honda, O. Kitao, H. Nakai, T. Vreven, J. A. Montgomery Jr, J. E. Peralta, F. Ogliaro, M. Bearpark, J. J. Heyd, E. Brothers, K. N. Kudin, V. N. Staroverov, T. Keith, R. Kobayashi, J. Normand, K. Raghavachari, A. Rendell, J. C. Burant, S. S. Iyengar, J. Tomasi, M. Cossi, N. Rega, J. M. Millam, M. Klene, J. E. Knox, J. B. Cross, V. Bakken, C. Adamo, J. Jaramillo, R. Gomperts, R. E. Stratmann, O. Yazyev, A. J. Austin, R. Cammi, C. Pomelli, J. W. Ochterski, R. L. Martin, K. Morokuma, V. G. Zakrzewski, G. A. Voth, P. Salvador, J. J. Dannenberg, S. Dapprich, A. D. Daniels, O. Farkas, J. B. Foresman, J. Cioslowski and D. J. Fox, Gaussian, Inc, Wallingford CT, 2010.

24 A. D. Becke, Phys. Rev. A, 1988, 38, 3098-3100.

25 C. Lee, W. Yang and R. G. Parr, Phys. Rev. B: Condens. Matter Mater. Phys., 1988, 37, 785-789.

26 J. P. Perdew, K. Burke and M. Ernzerhof, Phys. Rev. Lett., 1996, 77, 3865-3868.

27 Y. Zhao and D. G. Truhlar, Theor. Chem. Acc., 2008, 120, 215241.

28 A. V. Marenich, C. J. Cramer and D. G. Truhlar, J. Phys. Chem. B, 2009, 113, 6378-6396.

29 T. H. Dunning Jr, J. Chem. Phys., 1989, 90, 1007-1023.

30 K. A. Peterson and T. H. Dunning Jr, J. Chem. Phys., 2002, 117, 10548-10560.

31 N. W. Larsen and L. Schulz, J. Mol. Struct., 2009, 920, 30-39. 32 L. Pauling, The Nature of the Chemical Bond, Cornell University Press, New York, 3rd edn, 1959.

33 Y. Zhao, N. E. Schultz and D. G. Truhlar, J. Chem. Theory Comput., 2006, 2, 364-382.

34 L. Pauling, The Nature of the Chemical Bond, Cornell University Press, Ithaca, New York, 1960.

35 P. I. Nagy, J. Phys. Chem. A, 2012, 116, 7726-7741. 